\title{
A NANOMECHANICAL PROTEIN CONCENTRATION DETECTOR USING A NANO-GAP SQUEEZING ACTUATOR WITH COMPENSATED DISPLACEMENT MONITORING ELECTRODES
}

\author{
Won Chul Lee ${ }^{1,2)}$, Young-Ho Cho ${ }^{1)}$, and Albert P. Pisano ${ }^{2)}$ \\ ${ }^{1)}$ Digital Nanolocomotion Center, Korea Advanced Institute of Science and Technology \\ Daejeon REPUBLIC OF KOREA \\ ${ }^{2)}$ Berkeley Sensor and Actuator Center, University of California at Berkeley \\ Berkeley, California USA
}

\begin{abstract}
We present a new class of the protein concentration detector based on the mechanical stiffness measurement of protein-receptor layers in a squeezed nano-gap. Compared to the previous protein size detector, the present device reduces the distortion and uncertainty in the displacement measurement by adding an actuated nano-gap and reference electrodes. Compared to the conventional protein detectors based on electrochemical, optical, and optomechnical principles, the present device also offers simple, inexpensive, and high-precision protein detection. We design and fabricate the protein concentration detector using an electrothermal actuator and two nano-gaps with reference electrodes. In experimental study, we verify that the present protein detector measures the size of the proteins, streptaividin and m-antibiotin, as $12.1 \pm 2.3 \mathrm{~nm}$ and $13.2 \pm 3.3 \mathrm{~nm}$ at the measurement uncertainty of $\pm 1.1 \mathrm{~nm}$, respectively, while showing the concentration detection sensitivity of $2.88 \mathrm{~N} / \mathrm{m} / \mathrm{nM}$ in the m-antibiotin concentration range of $5 \sim 10 \mathrm{nM}$.
\end{abstract}

\section{INTRODUCTION}

High-precision protein detectors are actively studied for diagnosis and prognosis. [1] Previously, we presented the nanomechanical detection principle [2], where the actuator squeezes the protein-receptor layers in the nano-gap through a mechanical spring $\left(k_{l}\right)$. This nanomechanical principle offers simple, inexpensive, and high-precision protein detection [2] compared to electrochemical [3], optical [4,5], optomechancial [6], and mechanical $[\mathbf{7 , 8}]$ principles.

From the nanomechanical principle, we presented the protein size detector(Fig.1a) [2] based on the squeezed displacement measurement of $x_{2}(t)$ in Fig.2a. The previous device [2], however, was unable to measure the protein concentration due to a couple of problems: 1) the distortion in the time-dependent squeezed displacement $\left(x_{2}(t)\right.$ in Fig.2a) that should be corrected by the timedependent actuated displacement of $\left.x_{l}(t) ; 2\right)$ the displacement measurement uncertainty of $\pm 7.4 \mathrm{~nm}[2]$ caused by signal drift and noise.

In this work, we succeed to measure the protein concentration by solving the above-mentioned problems as follows: 1) We add an actuated nano-gap(Fig.1b) to measure $x_{I}(t)$ in addition to $x_{2}(t)$. We synchronize $x_{1}(t)$ and $x_{2}(t)$ to obtain the directed correlation(Fig. $2 \mathrm{~b}$ ) between $x_{1}$ and $x_{2}$, thus eliminating the distortion included in $x_{I}(t)$ and $x_{2}(t)$; 2) We use reference electrodes (Fig. 3) in both actuated and squeezed nano-gaps to compensate the signal drift and noise, thus reducing the displacement measurement uncertainty from $\pm 7.4 \mathrm{~nm}$ to $\pm 1.1 \mathrm{~nm}$. We also experimentally verify the selectivity, repeatability, and sensitivity of the protein concentration detector.
Figure $2 \mathrm{~b}$ illustrates the protein detection principle of the device, where the mechanical stiffness of protein-receptor layers is measured by $x_{1}-x_{2}$ relationship. When the squeezing electrode touches the receptor-protein layers on the nano-gap surfaces (Fig.3), the layers provide added stiffness ( $k_{r p}$ in Fig.1) to the mechanical spring $\left(k_{1}\right)$. The added stiffness changes the slope of the modulation lines on $x_{1}-x_{2}$ plane(Fig.2b). We measure the protein concentration from the slope $(s)$ of the modulation line, while detecting the protein presence and size from the ordinate shift(Fig.2a) of the slope changing point $(P)$.

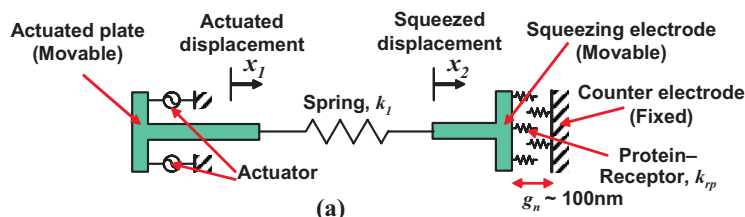

(a)

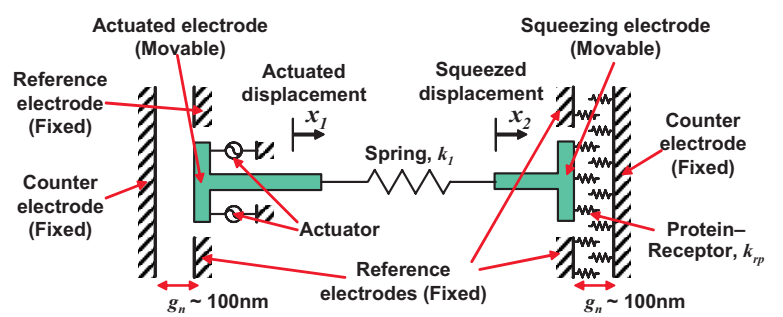

(b)

Figure 1. Comparison of the nanomechanical protein detectors: (a) the previous protein presence detector [2]; (b) the present protein concentration detector, measuring the actuated and squeezed displacements using compensated displacement monitoring electrodes.

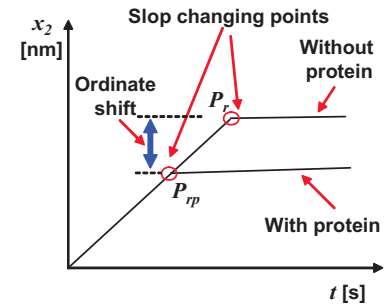

(a)

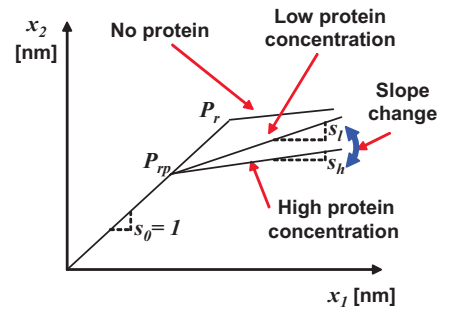

(b)
Figure 2. Working principle of the nanomechanical protein detectors: (a) the previous protein presence detection [2] using the relationship between the actuation time, $t$, and the squeezed displacement, $x_{2}$; (b) the present protein concentration detection using the relationship between the actuated and squeezed displacements, $x_{1}$ and $x_{2}$. 


\section{DESIGN AND FABRICATION}

We design the nanomechanical protein detector as shown in Figure 4 and Table 1. We use an electrothermal actuator and the nano-gaps for the operation in a buffer solution. The nano-gap, $g_{n}$, is formed from a fabricated initial gap, $g_{i}$. We obtain the nanoscale distance between the squeezing electrode and the counter electrode after attaching the counter electrode to stoppers.

Figure 4 contains a top view of the electrothermal actuator. The currents though heating bars generate heat, elongating them and deforming the hinges. Thus, the output port $\left(m_{1}\right)$ of the electrothermal actuator moves in the $x_{1}$-direction. From 2dimensional electrothermal and thermomechanical analysis, we obtain the electrothermal actuation of $699 \mathrm{~nm}$ at the temperature change of $29.6^{\circ} \mathrm{C}$. We can verify that the displacement of the electrothermal actuator is large enough to cover the nano-gap of $100 \mathrm{~nm}$.

We can detect the squeezed motion, $x_{2}$, using an impedance change of the nano-gap. The squeezed motion changes the impedance of the buffer solution between the squeezing electrode and the counter electrode. In order to reduce the displacement measurement uncertainty, we use reference electrodes (Fig. 3) in both actuated and squeezed nano-gaps to compensate the signal drift and noise.

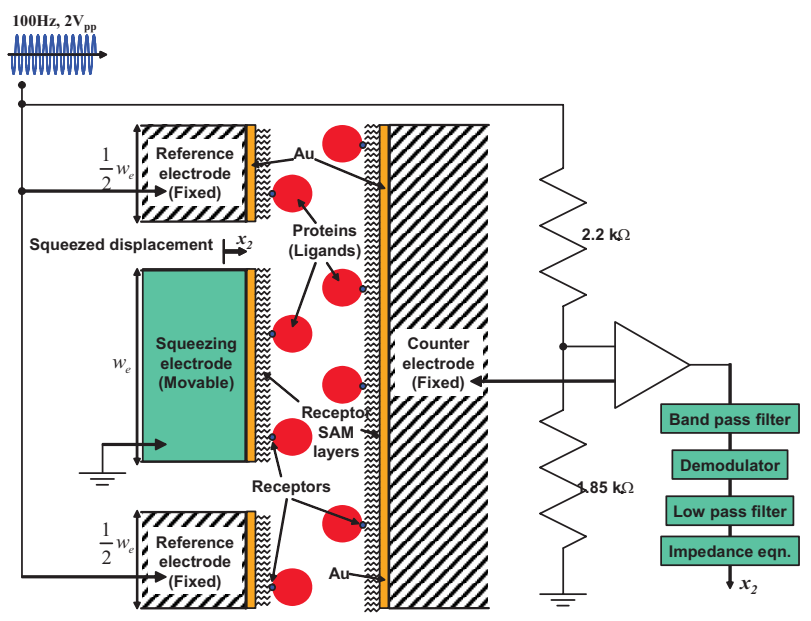

Figure 3. Squeezed and reference nano-gaps and compensated displacement monitoring methods in the protein concentration detector.

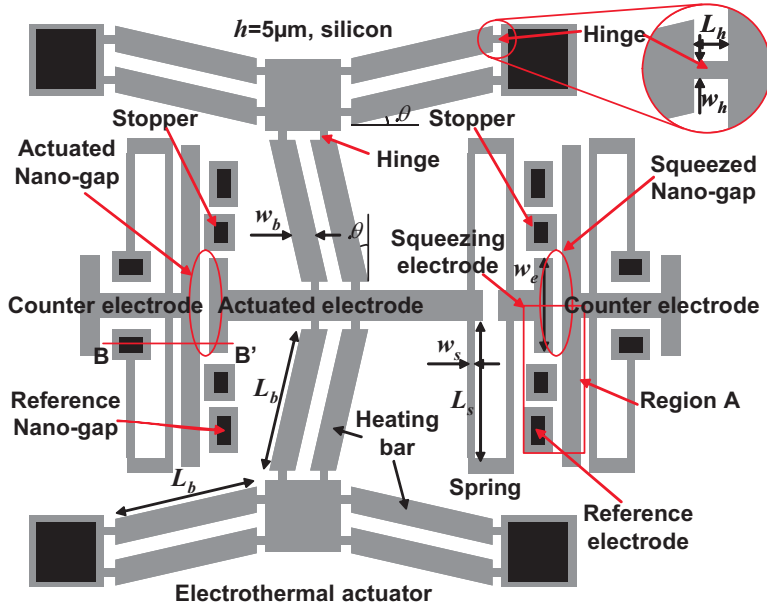

Figure 4. Top view of the protein concentration detector.

Table 1. Measured dimensions of the fabricated devices

\begin{tabular}{c|c}
\hline Structures* & Dimensions \\
\hline \hline Structure thickness, $h$ & $5 \mu \mathrm{m}$ \\
\hline Bar length, $L_{b}, \times$ Bar width, $w_{b}$ & $220 \mu \mathrm{m} \times 28 \mu \mathrm{m}$ \\
\hline Bar angle, $\theta_{b}$ & $6^{\circ}$ \\
\hline Hinge length, $L_{h}, \times$ Hinge width, $w_{h}$ & $10.0 \mu \mathrm{m} \times 4.0 \mu \mathrm{m}$ \\
\hline Spring length, $L_{s}, \times$ Spring width, $w_{s}$ & $200 \mu \mathrm{m} \times 3.2 \mu \mathrm{m}$ \\
\hline Electrode width, $w_{e}$ & $52.0 \mu \mathrm{m}$ \\
\hline Initial gap, $g_{i}$ & $10.0 \mu \mathrm{m}$ \\
\hline Nano-gap, $g_{n}$ & $100 \mathrm{~nm}$ \\
\hline
\end{tabular}

*The structures are shown in Figs. 3 and 4.

The designed protein concentration detector is fabricated (Fig.5) as shown in Fig.6, followed by the process of receptor immobilization. The two-mask microfabrication process is shown in Figure 5, representing the cross section along B-B' in Fig.4. Nano-gap surface has been smoothed by oxidation(Fig.5b) and oxide-etching(Fig.5c) during the fabrication process.

Biotin, the receptor, is immobilized on the gold surface of the nano-gap electrodes using SAM (Self Assemble Monolayer). We deposit the mixed SAM layer, composed of 11mercaptoundecanol and 16-mercaptohexa-decanoic acid. Then, the gold surface is biotinylated using biotinamidohexanoic acid hydrazide. Figure 7 shows the fluorescent image, which indicates that the biotin is immobilized on the nano-gap electrodes.

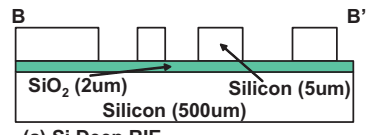

(a) Si Deep RIE

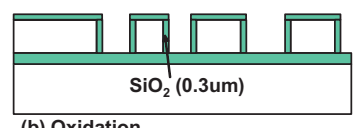

Figure 5. Microfabrication process showing the cross section along $B$ - $B$ ' in Fig. 4 .

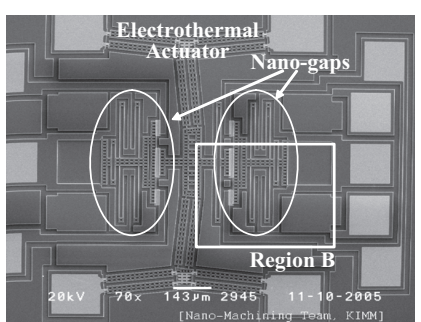

(a)
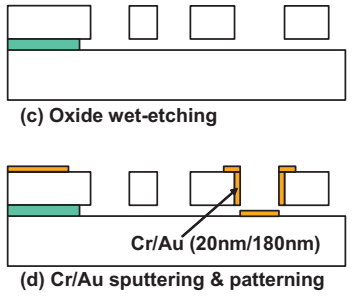

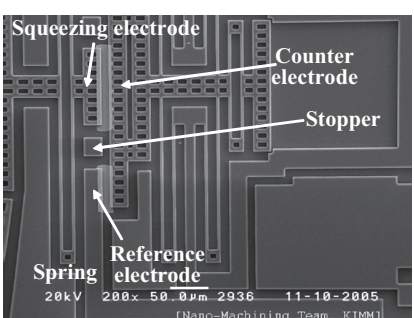

(b)

Figure 6. SEM photographs of the protein concentration detector: (a) overall structure; (b) an enlarged view of the Region B in (a).

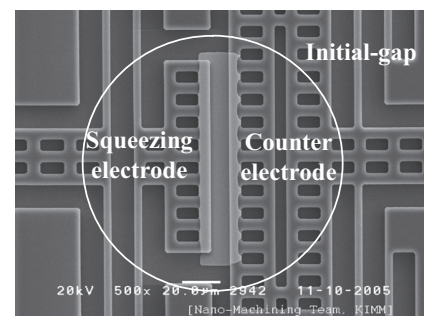

(a)

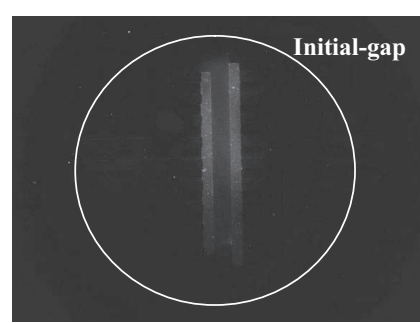

(b)
Figure 7. Microscope images of the initial-gap in the protein concentration detector with FITC tagged streptavidin: (a) SEM image; (b) fluorescent microscope image. 


\section{EXPERIMENTAL RESULTS}

In experimental study(Table 2), we characterize 1) the distortion in the time-dependent squeezed displacement, 2) the displacement measurement uncertainty, 3) the selectivity, 4) the repeatability, and 5) the sensitivity of the protein concentration detector. 1) The distortion, expressed by the $t-x_{2}$ nonlinearity $(3.0 \%)$ in Fig.8, is eliminated by the time-dependent actuated displacement of $x_{l}(t)$ in Fig.8a. 2) The displacement measurement uncertainty are reduced to $\pm 1.1 \mathrm{~nm}$ (Fig. 8 ) due to the compensated displacement monitoring electrodes. 3) The protein detection selectivity is verified by Fig.9 and Table 3: The detector generates the size detection signal $(12.1 \pm 2.3 \mathrm{~nm})$ for receptoractive protein and the null detection signal for receptor-inactive protein. 4) The detection repeatability is demonstrated by Fig. 10 and Table 4: The detector measures the protein size $(13.2 \pm 3.3 \mathrm{~nm})$. After the protein removal, the size detection signal returns to the initial state (null signal) indicating no protein.

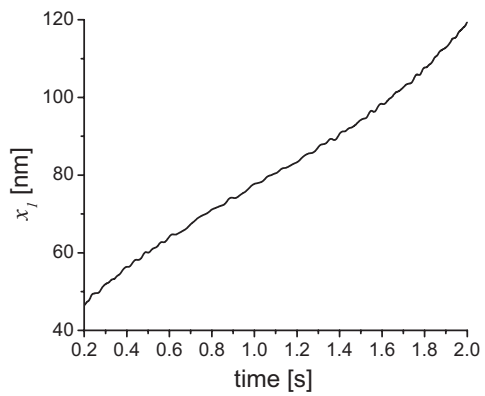

(a)

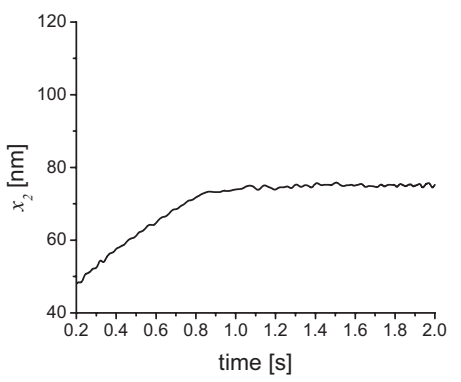

(b)

Figure 8. Measured displacements of the protein concentration detector: (a) the actuated displacement, $x_{1} ;$ (b) the squeezed displacement, $x_{2}$

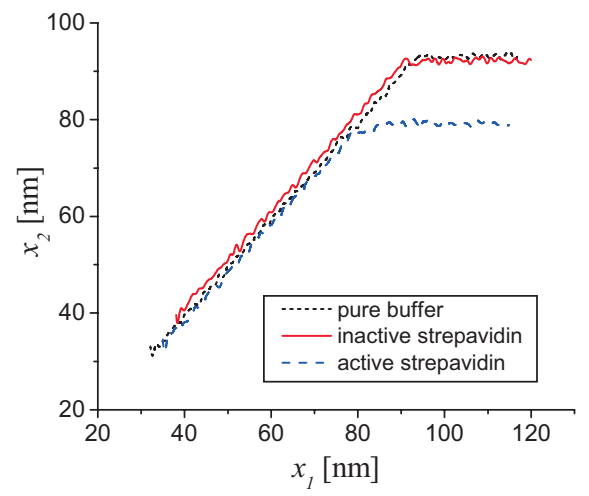

Figure 9. Experimental squeezed displacement, $x_{2}$, for the actuated displacement, $x_{1}$, in the selectivity verification using receptor-active and receptor-inactive proteins.
Table 2. Receptors and proteins used in the experiments for the performance verification

\begin{tabular}{c|c|c}
\hline Verified performance & Receptor & Protein \\
\hline \hline Selectivity & Biotin & Streptavidin \\
\hline Repeatability & Biotin & M-antibiotin \\
\hline Sensitivity & Biotin & M-antibiotin \\
\hline
\end{tabular}

Table 3. Experimental ordinate shifts* of the slope changing points for the selectivity verification using 3 devices, each from different dice

\begin{tabular}{c|c|c}
\hline Device \# & Inactive streptavidin** & Active streptavidin \\
\hline \hline $\mathrm{A}$ & $2.3 \pm 2.1 \mathrm{~nm}$ & $12.1 \pm 2.4 \mathrm{~nm}$ \\
\hline $\mathrm{B}$ & $-0.3 \pm 2.9 \mathrm{~nm}$ & $10.9 \pm 2.9 \mathrm{~nm}$ \\
\hline $\mathrm{C}$ & $1.1 \pm 2.9 \mathrm{~nm}$ & $13.2 \pm 3.1 \mathrm{~nm}$ \\
\hline \hline Average & $1.0 \pm 2.6 \mathrm{~nm}$ & $12.1 \pm 2.3 \mathrm{~nm}$ \\
\hline
\end{tabular}

*The ordinate shifts, obtained from the five repeated measurements of the slope changing points, are indicated in the forms of (average $\pm 2 \sigma$ ).

$* * 0.5 \mathrm{uM}$ streptavidin solution is inactivated by the pre-incubation with

2.0uM d-biotin solution.

Table 4. Experimental ordinate shifts* of the slope changing points for the repeatability verification using 3 devices, each from different dice

\begin{tabular}{c|c|c}
\hline Device \# & M-antibiotin addition & M-antibiotin removal \\
\hline \hline A & $13.9 \pm 3.2 \mathrm{~nm}$ & $-2.3 \pm 3.0 \mathrm{~nm}$ \\
\hline $\mathrm{B}$ & $14.3 \pm 2.7 \mathrm{~nm}$ & $0.1 \pm 2.5 \mathrm{~nm}$ \\
\hline $\mathrm{C}$ & $11.3 \pm 1.2 \mathrm{~nm}$ & $1.0 \pm 1.3 \mathrm{~nm}$ \\
\hline \hline Average & $13.2 \pm 3.3 \mathrm{~nm}$ & $-0.4 \pm 3.4 \mathrm{~nm}$ \\
\hline
\end{tabular}

*The ordinate shifts, obtained from the five repeated measurements of the

slope changing points, are indicated in the forms of (average $\pm 2 \sigma$ ).

Table 5. Experimental slopes and receptor-protein stiffness for different concentrations of m-antibiotin solution

\begin{tabular}{c|c|c|c|c|c}
\hline \multirow{2}{*}{$\begin{array}{c}\text { Concen- } \\
\text { tration }\end{array}$} & \multicolumn{4}{|c|}{ Slope, $s$} & $\begin{array}{c}\text { Stiffness of } \\
\text { receptor-protein } \\
\text { layers, } n \cdot k_{r p}\end{array}$ \\
\cline { 2 - 6 } & Device A & Device B & Device C & Ave. $\pm 2 \sigma$ & N/A \\
\hline \hline $1 \mathrm{nM}$ & N/A & N/A & N/A & N/A & N/A \\
\hline $3 \mathrm{nM}$ & N/A & N/A & N/A & N/A & $9.6 \pm 4.6 \mathrm{~N} / \mathrm{m}$ \\
\hline $5 \mathrm{nM}$ & 0.228 & 0.171 & 0.240 & $0.213 \pm 0.074$ & 9.07 .023 \\
\hline $7 \mathrm{nM}$ & 0.128 & 0.151 & 0.137 & $0.139 \pm 0.023$ & $15.8 \pm 3.0 \mathrm{~N} / \mathrm{m}$ \\
\hline $10 \mathrm{nM}$ & 0.104 & 0.095 & 0.088 & $0.096 \pm 0.016$ & $24.1 \pm 4.5 \mathrm{~N} / \mathrm{m}$ \\
\hline $20 \mathrm{nM}$ & 0.095 & 0.100 & 0.109 & $0.101 \pm 0.015$ & $22.6 \pm 3.5 \mathrm{~N} / \mathrm{m}$ \\
\hline $500 \mathrm{nM}$ & 0.101 & 0.083 & 0.107 & $0.097 \pm 0.025$ & $23.8 \pm 7.2 \mathrm{~N} / \mathrm{m}$ \\
\hline
\end{tabular}

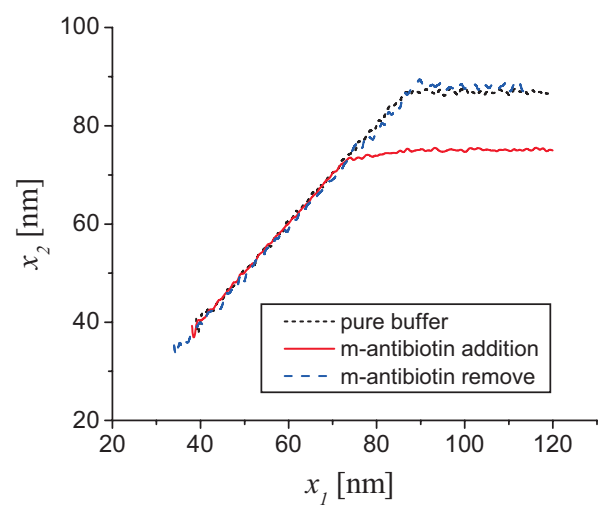

Figure 10. Experimental squeezed displacement, $x_{2}$, for the actuated displacement, $x_{1}$, in the repeatability verification using $m$ - antibiotin. 
5) We verify the sensitivity of the fabricated device from Table 5, where the slope and the stiffness are obtained from the modulation lines (Fig.11) for protein-receptor layers in the protein concentration range of $1 \sim 500 \mathrm{nM}$. For the protein concentration of $5 \sim 10 \mathrm{nM}$ (Fig.12), the $\operatorname{stiffness}\left(n \cdot k_{r p}\right)$ is measured in the range of $9.6 \sim 24.1 \mathrm{~N} / \mathrm{m}$ at the sensitivity of $2.88 \mathrm{~N} / \mathrm{m} / \mathrm{nM}$. The stiffness is saturated over $10 \mathrm{nM}$ concentration, indicating the protein-receptor binding saturation.

It is experimentally verified that the present protein detector measures the size of the proteins, streptaividin and m-antibiotin, as $12.1 \pm 2.3 \mathrm{~nm}$ and $13.2 \pm 3.3 \mathrm{~nm}$ at the measurement uncertainty of $\pm 1.1 \mathrm{~nm}$, respectively, while showing the concentration detection sensitivity of $2.88 \mathrm{~N} / \mathrm{m} / \mathrm{nM}$ in the m-antibiotin concentration range of $5 \sim 10 \mathrm{nM}$.

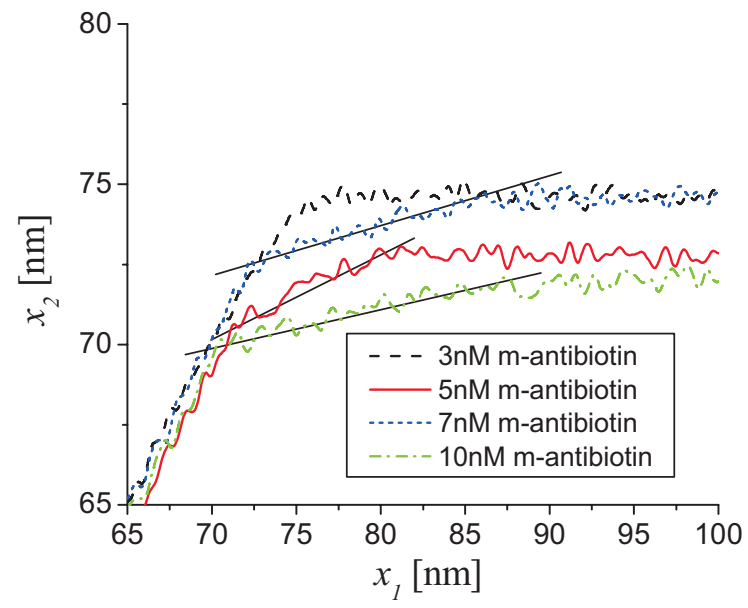

Figure 11. Experimental squeezed displacement, $x_{2}$, for the actuated displacement, $x_{1}$, in various m-antibiotin concentrations.

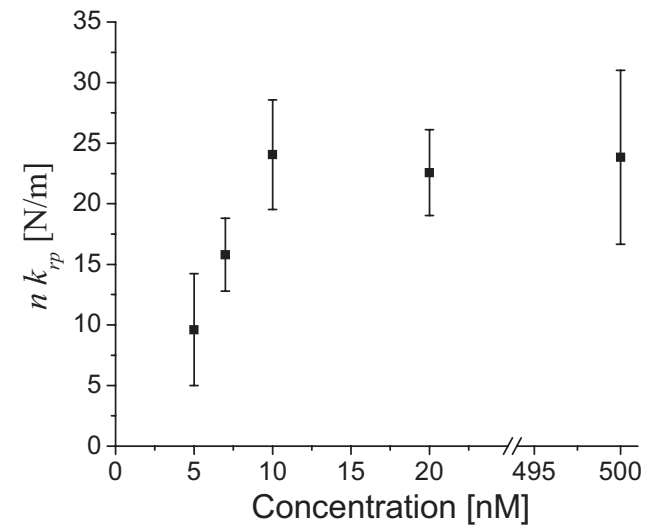

Figure 12. Experimental stiffness of receptor-protein layers, $n \cdot k_{r p}$, in various m-antibiotin concentrations.

\section{CONCLUSIONS}

We presented a new class of the protein concentration detector based on the mechanical stiffness measurement of protein-receptor layers in a squeezed nano-gap. Compared to the previous protein size detector based on the squeezed displacement measurement, we added the actuated nano-gap and reference electrodes. Thus, the present device compensated the distortion in the time-dependent squeezed displacement and reduced the displacement measurement uncertainty of $\pm 7.4 \mathrm{~nm}$ caused by signal drift and noise. We designed and fabricated the protein concentration detector using the electrothermal actuator and two nano-gaps with reference electrodes. In experimental study, we verified that the present protein detector measured the size of the proteins, streptaividin and m-antibiotin, as $12.1 \pm 2.3 \mathrm{~nm}$ and $13.2 \pm 3.3 \mathrm{~nm}$ at the measurement uncertainty of $\pm 1.1 \mathrm{~nm}$, respectively, while showing the concentration detection sensitivity of $2.88 \mathrm{~N} / \mathrm{m} / \mathrm{nM}$ in the m-antibiotin concentration range of $5 \sim 10 \mathrm{nM}$.

\section{ACKNOWLEDGEMENTS}

This work has been supported by the National Creative Research Initiative Program of the Ministry of Science and Technology (MOST) under the project title of "Realization of BioInspired Digital Nanoactuators."

\section{REFERENCES}

[1] S. Hanash, "Disease Proteomics," Nature, Vol.422 (2003), pp.226-232. [2] W. C. Lee and Y.-H. Cho, "Nanomechanical Protein Detectors Using Electrothermal Nano-gap Actuators," MEMS'04 (2004), pp.629-632.

[3] M. Lambrechts and W. Sansen, Biosensors: Microelectrochemical Devices, IOP Publishing Ltd., Philadelphia and New York, 1992.

[4] R.P. Ekins, "Ligand Assays: from Electrophoresis to Miniaturized Microarrays," Clinical Chemistry, Vol.44, No.9 (1998), pp.2015-2030.

[5] J. Homola, S.S. Yee and G. Gauglitz, "Surface Plasmon Resonance Sensors: Review," Sensors and Actuators, Vol.B54 (1999), pp.3-15.

[6] J. Fritz, M. K. Baller, H. P. Lang, H. Rothuizen, P. Vettiger, E. Meyer, H.-J. Güntherodt, Ch. Gerber, and J. K. Gimzewski, "Translating Biomolecular Recognition into Nanomechanics," Science, Vol.288, No.5464 (Apr. 14, 2000), pp.316-318.

[7] J. Zhang and E.S. Kim, "Vapor and Liquid Mass Sensing by Micromachined Acoustic Resonator," Proc. 16th IEEE Inter. Conf. Micro Electro Mechanical Systems (MEMS 2003), Kyoto Inter. Conference Hall, Kyoto, Japan (Jan. 19-23, 2003), pp.470-473.

[8] J. Rickert, A. Brecht and W. Gopel, "QCM Operation in Liquids: Constant Sensitivity during Formation of Extended Protein Multilayers by Affinity," Analytical Chemistry, Vol.69, No.7 (1997) pp.1441-1448. 